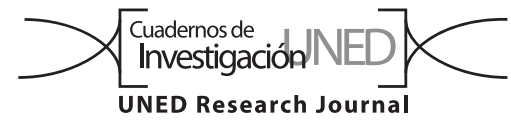

\title{
Influencia del pez León (Pterois miles y Pterois volitans) en la cadena de valor de la pesca artesanal, Caribe sur de Costa Rica
}

\author{
Max Laguna Cruz', José Pereira-Chaves ${ }^{2,3}$, Roger Ríos Duarte ${ }^{4}$ \\ 1. Universidad Estatal a Distancia, Programa de Manejo de Recursos Naturales, 2050 San José, Costa Rica; mlagunac6@gmail.com, \\ (iD) https://orcid.org/0000-0003-2226-830X \\ 2. Universidad Nacional, Escuela de Ciencias Biológicas, Heredia, Costa Rica. \\ 3. Universidad Estatal a Distancia, Escuela de Ciencias Exactas y Naturales, 2050 San José, Costa Rica; jose.pereira.chaves@una.cr, \\ (D) https://orcid.org/0000-0001-6056-3364 \\ 4. Universidad Estatal a Distancia, 2050 San José, Costa Rica; roger.riosd@gmail.com,
}

(D) https://orcid.org/0000-0002-0148-2721

Recibido 15-I-2019 • Corregido 19-III-2019 • Aceptado 03-IV-2019

DOI: https://doi.org/10.22458/urj.v11i3.2267

\begin{abstract}
Lionfish (Pterois miles and Pterois volitans) influence on the artisanal fishing value chain, Southern Caribbean of Costa Rica". Introduction: The "Lionfish" are actually two invasive species that occur in several parts of the world, and their effect on local fisheries needs study. Objective: To analyze the influence of these fish on the artisanal fisheries value chain in the Southern Caribbean of Costa Rica. Methods: We surveyed 181 fishermen, restaurant owners and resident consumers, and made counts during fishing operations. Results: Lionfish do not reach consumers in significant numbers, mainly because there is not enough initial input of raw material to compete against established species. Conclusion: we recommend a campaign for the consumption of Lionfish in Costa Rica.
\end{abstract}

Key words: Lionfish, value chain, artisanal fisheries, Southern Caribbean, Costa Rica.
RESUMEN: Introducción: Comúnmente se llama "pez León" a dos especies invasoras que se encuentran en varias partes del mundo, y su efecto sobre la pesca local necesita estudio. Objetivo: Analizar la influencia de estos peces en la cadena de valor de la pesca artesanal en el Caribe sur de Costa Rica. Métodos: Encuestamos a 181 pescadores, dueños de restaurantes y consumidores residentes, e hicimos recuentos durante las operaciones de pesca. Resultados: El pez León no llega a los consumidores en cantidades significativas, principalmente porque no hay suficiente aporte inicial de materia prima para competir contra las especies establecidas. Conclusión: Recomendamos una campaña para el consumo de pez León en Costa Rica.

Palabras Claves: Pez León, cadena de valor, pesca artesanal, Caribe sur, Costa Rica.
Durante la última década, al pez León (Pterois miles y Pterois volitans), se le ha dado reputación de especie invasora por impactar los ecosistemas, acabando con las especies nativas, poniéndose en riesgo las actividades pesqueras e impactando a toda la cadena de valor, lo que ha motivado a que se realicen investigaciones en el país para documentar la realidad del impacto que pueda ocasionar esta especie exótica en una cadena de valor del Caribe sur.

El experimento de Albins y Hixon (2008) ha sido uno de los más controversiales, dado el impacto ecológico que ocasionó el pez León, en los arrecifes de las Bahamas, donde se redujo significativamente el reclutamiento de los peces nativos en un $79 \%$ durante cinco semanas y en virtud del efecto sobre la cadena alimenticia sugieren que ya está teniendo impactos negativos sustanciales en otros arrecifes de coral del Atlántico, sin tomar en cuenta que los corales son distintos en otros sitios.

Posteriormente, dos estudios en las Bahamas vincularon al pez León con una reducción de biomasa de peces y de diversidad en los arrecifes de coral, que en este último caso causó la eutroficación por la dominación de las algas (Lesser \& Slattery, 2011; Green, Akins, Maljković, \& Côté, 2012).

Progresivamente, se han recolectado datos sobre variedad de hábitats, tolerancia de alto rango de profundidad, temperatura y salinidad en México y Estados Unidos y a partir de 2008, varios estudios científicos exigían 
acciones en forma de extracciones para controlar las poblaciones de pez León, llamadas que se hicieron eco en las agencias gubernamentales con responsabilidades en áreas marinas protegidas en México (Comisión Nacional de Áreas Naturales Protegidas, CONANP) y en los Estados Unidos (NOAA), que han dirigido los esfuerzos para una estrategia de gestión regional (Morris, 2013).

Karp et al. (2015) incentivaron la eliminación del pez León, por medio de promover actividades como las artesanías, usando las espinas y las aletas del pez León. En Belice, Bahamas, San Vicente y Las Granadinas, se logró aumentar las ganancias de los pescadores artesanales en un $40 \%$ y a su vez ofreció una oportunidad económica a las mujeres.

En las Islas Caimán, Johnson y McCoy (2015) señalan que el Departamento de Medio Ambiente promovió los torneos para involucrar al público en los esfuerzos de remoción del pez León y en 2014 estos esfuerzos, se complementaron con la comercializaron, por medio de una empresa con licencia para cosecharlo para la exportación. De esto, se concluye como valioso el aporte de estos programas para su remoción.

Por su parte, Sandel (2011) estudió el estado de la población en el Caribe sur de Costa Rica. Para ello muestreó cuatro sitios, una vez por semana, durante los meses de marzo a junio del 2011, los censos se realizaron utilizando equipo snorkel hasta $7 \mathrm{~m}$ de profundidad y se contaron todos los especímenes que se pudieran ver dentro de cada transecto. Se demostró que las poblaciones tuvieron una densidad promedio poblacional de 91 individuos/Ha en dicho año.

Galarza y Kamiche (2015) utilizaron el modelo de cadenas de valor, de la Organización de las Naciones Unidas para la Alimentación y la Agricultura (2014) para identificar problemas y recomendaciones para la especie anchoveta en Perú, esta se basa en incluir todas las etapas, desde la producción de materia prima (captura), pasando por la transformación y comercialización del producto hasta llegar al usuario final (consumidor). Se concluyó que a pesar de que los pescadores artesanales dan inicio a la cadena de valor, son los que menos se benefician. Siendo un modelo similar a la metodología que instituye esta investigación.

Con la incursión del pez León por el Atlántico, algunos países se motivan a realizar acciones conjuntas, Carballo (2015) considera que el discurso dominante cataloga a esta especie como una amenaza, por lo tanto, mala para los ecosistemas, basándose en los impactos observados. Algunos científicos sociales criticaron la práctica de apelar al factor miedo mediante el uso de metáforas de militaristas y catastróficas relacionando la especie como invasora y amenazadora, dando como tal un sentido de urgencia y necesidad de acción para la reconstrucción de los stocks de peces comerciales y la conservación de arrecifes, lo que hace evidente la necesidad de un abordaje integral del estudio del pez León, donde se considera además de factores biológicos, ecológicos, otros como el cambio climático, la sobrepesca y la plataforma continental.

En 2016 la Presidencia de la República de Costa Rica vía decreto crea la "Comisión Nacional para el Manejo, Control y Aprovechamiento del pez León en Costa Rica". Allí indica que está siendo capturado por el 50\% de las nasas de los pescadores artesanales del Caribe sur, declara de nacional su manejo y aprovechamiento, e insta a entidades públicas y privadas para que contribuyan con aporte económico, logístico y técnico para su manejo (Poder Ejecutivo, 2016).

Aquí analizamos su influencia en la cadena de valor de la pesca artesanal en el Caribe sur de Costa Rica.

\section{MATERIALES Y MÉTODOS}

El estudio se realizó entre mayo y octubre del 2016, en la costa Caribe sur de Costa Rica, específicamente en los pueblos de Cieneguita, Cahuita, Punta Uva, Puerto Viejo y Manzanillo, además abarcó alrededor de cinco millas náuticas mar adentro desde estos pueblos (Fig. 1).

A lo largo de esta línea costera se encuentra el Parque Nacional Cahuita con un área de 22,4Ha de área marina protegida (Sistema Nacional de Áreas de Conservación, 2016) y el Refugio Nacional de Vida Silvestre Gandoca Manzanillo (REGAMA) humedal con declaratoria de importancia internacional (sitio RAMSAR) dada la gran biodiversidad que alberga estos ecosistemas marinos, con $4436 \mathrm{Ha}$ de área marina protegida (Parques Nacionales de Costa Rica, 2016).

Para describir la cadena de valor de pesca artesanal establecida tradicionalmente antes de la llegada del pez León al Caribe sur, se estableció con base en aquellos productos pesqueros que han alcanzado completar el ciclo de la cadena de valor en el tiempo, especies usadas en el libro de cocina tradicional costarricense del Ministerio de Cultura y Juventud y del Centro de Investigación y Conservación del Patrimonio Natural, presenta los productos pesqueros que se encuentran en la cocina Caribeña de Costa Rica, los cuales son la corvina, macarela, camarones, langosta y pargo (Álvarez, 2014). A su vez, Ramos (2016) funcionario del instituto costarricense de pesca y acuicultura (INCOPESCA) aporta los volúmenes de capturas de estos productos y se aplicó una guía 


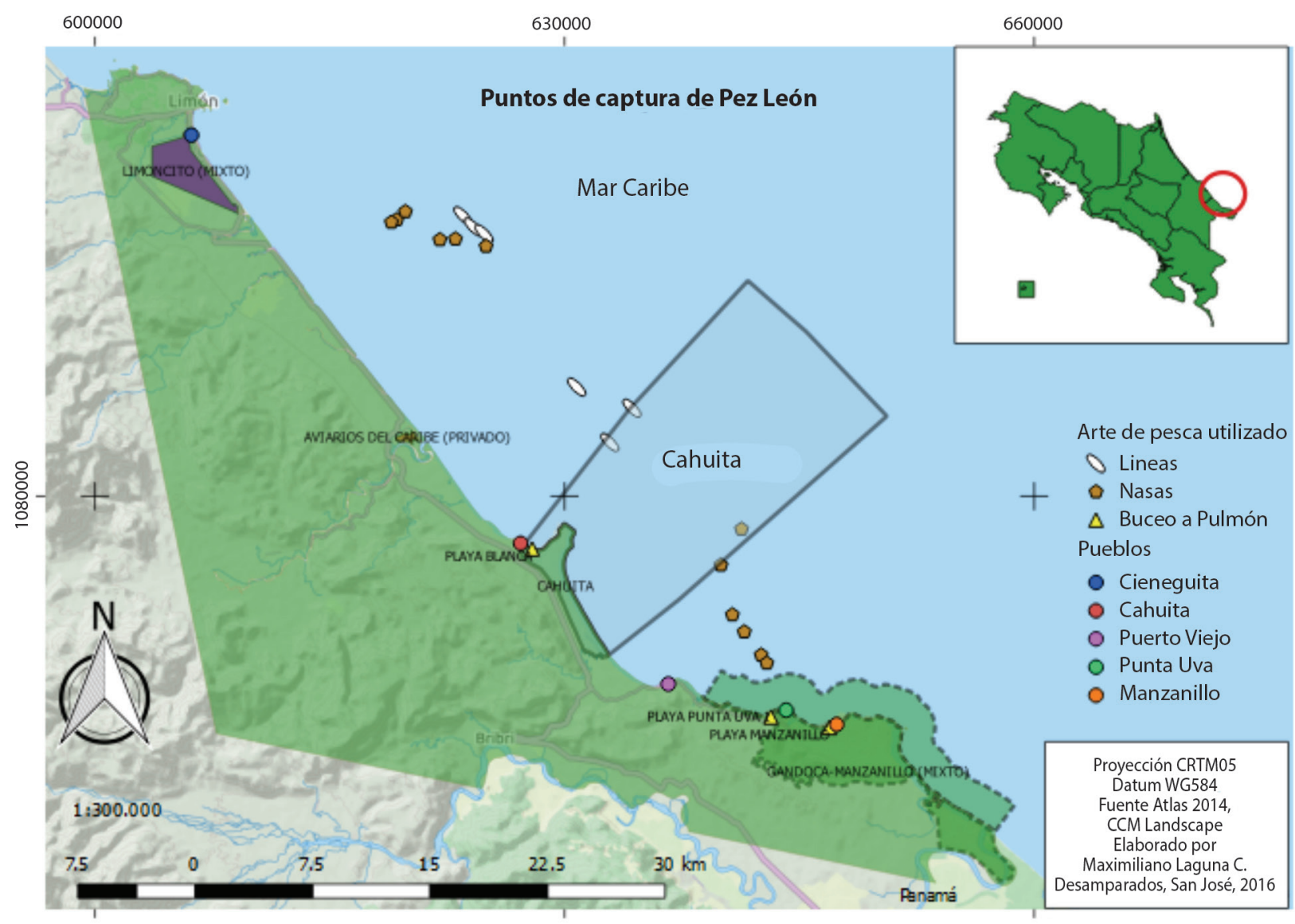

Fig. 1. Zona de estudio.

de preguntas a la Asociación de pescadores del litoral Atlántico (ASPELTA) para conocer los precios y las artes de pesca utilizada para capturarlos, así como la gastronomía que ofrecen los restaurantes para el consumidor.

Un arte de pesca es el conjunto de materiales utilizados para la extracción del recurso pesquero (Ustate, 2005). En el Caribe sur de Costa Rica las artes de pesca artesanal más utilizadas son: las Nasas, Línea y buceo a pulmón (Mairena, 2015; McDonald, 2015).

Para capturar el Pez León se colocaron 12 parejas de nasas, distribuidas desde Cieneguita hasta Puerto Viejo, las mismas eran rectangulares de $1,5 \mathrm{~m}$ de largo por $1 \mathrm{~m}$ de ancho, hecho con una estructura de madera y envuelta con una red metálica. Cada nasa se sumergió a una profundidad entre 30 y 60 metros durante 24 horas en el fondo marino. Con el arte de pesca línea, se colocaron seis en el área que va desde Cieneguita hasta Cahuita por un espacio de tres horas. Estas líneas contenían una línea principal horizontal de $120 \mathrm{~m}$ de largo con alrededor de 90 anzuelos con trozos de macarela, la línea horizontal principal quedó en la parte media de la columna del agua a unos $40 \mathrm{~m}$ de profundidad aproximadamente.
Por otra parte, el buceo a pulmón es una antigua práctica que los pescadores caribeños utilizan como parte de su cultura para extraer productos del mar, en esta investigación fue de acuerdo a la capacidad pulmonar de tres buceadores teniendo una media de inmersión de $10 \mathrm{~m}$ de profundidad, para llegar a estas profundidades se nadó cerca de $400 \mathrm{~m}$ desde la costa con ayuda de snorkel, en un tiempo total de dos horas para explorar y capturar Pez León desde el litoral hasta las crestas de arrecife. Los sitios de buceo a pulmón se llevaron a cabo en las áreas marinas protegidas de Cahuita, Punta Uva y Manzanillo.

En cada arte de pesca del pez León utilizado, se registró el número de individuos y peso total de la pesca, Además, los sitios fueron elegidos por los pescadores de acuerdo con las zonas donde aseguran haberlo capturado anteriormente y con las mismas artes de pesca. Estos sitios representan la vida real cotidiana donde se realiza la faena de captura del pez León en el Caribe sur, sitio donde se ha establecido esta especie (Sandel, 2011).

Los pescadores están agrupados en tres asociaciones con relación a su zona de pesca, así los que pescan frente a la costa de Cieneguita están concentrados en 
la "Asociación de Pescadores del Litoral Atlántico". Los que pescan en los alrededores de Cahuita conforman la "Asociación de Pescadores de subsistencia y acuicultura del Caribe sur de Cahuita" (Hernández, 2015). Los que pescan en los alrededores de Puerto Viejo y Manzanillo conforman la "Asociación de Pescadores Artesanales del Caribe sur" (Taylor, 2015). Esta última fue la que promovió la creación del "protocolo para la Captura, extracción y disposición final del pez león en Costa Rica, también esta asociación promueve los torneos de PL en el Caribe sur.

Se aplicó una encuesta a 43 pescadores representantes de cada asociación, la selección de la muestra se realizó de forma aleatoria por medio de visitas a los lugares donde habita el pez León: Cieneguita, Cahuita, Puerto Viejo y Manzanillo, con el fin de distribuir homogéneamente la muestra en la zona donde el pez León habita. Cada asociación cuenta con alrededor de 60 pescadores para un total de 180 (Ríos, 2015), en el centro de acopio de Cieneguita los pescadores llegan con productos como el pargo, macarela, camarones, langosta, greenjack, jurel, pez chancho y bolillo (Parkins, 2015).

De acuerdo con Lid, Marchal y Wathen (2012), la fórmula para establecer una muestra representativa de poblaciones finitas y que se desconoce la varianza es la siguiente:

$$
n o=\pi(1-\pi)\left(\frac{Z}{e}\right)^{2}
$$

Donde

$\mathrm{n}=180$ pescadores

$\pi=$ estimación de la varianza con 0,7 ; las condiciones de trabajo son similares para todos los trabajadores, se prevé que no hay alta variabilidad en las opiniones

$Z=1,645$ valor de la distribución normal estándar con $90 \%$ de confianza

e $=$ error máximo permitido por el investigador 0,10

$$
n o=0,7(1-0,7)\left(\frac{1,645}{0,1}\right)^{2} n o=57
$$

Ajustando el factor de corrección

$$
n 1=\frac{n o}{1+\frac{n o}{N}} n 1=\frac{57}{1+\frac{57}{180}} n 1=43
$$

A su vez, se aplicaron 38 encuestas a los dueños o encargado de los restaurantes que se ubican en las costas del Caribe sur distribuidos desde Cieneguita; Cahuita, Puerto Viejo y Manzanillo, para determinar si ha habido un cambio en la oferta gastronómica, debido a la introducción del pez León, con base en los productos tradicionales del libro de cocina caribeña (Álvarez, 2014), al ser una zona turística se amplía la oferta gastronómica.

Se aplicaron 100 encuestas a los consumidores que viven en el área de estudio, para determinar si habido algún cambio en su dieta de pescado en estos últimos años. El propósito fue conocer la tendencia que se evidencio en la zona.

La selección de la muestra se realizó al azar entre las que asistieron a la feria del agricultor en los lugares: Cieneguita, Cahuita y Puerto Viejo, con el fin de distribuirla lo más uniforme posible y dada la facilidad que se presenta al concentrar una gran cantidad de personas representantes de cada pueblo. Además, los encuestados debían cumplir la condición de no ser pescadores y ser mayores de 18 años, ya que tienen mayor noción de los acontecimientos sucedidos desde la llegada del pez León en el 2009.

Por otra parte, el concepto de cadena de valor lo introduce Porter (2005), quien señala que el mismo radica en lograr la fluidez de los procesos centrales de una empresa, lo cual implica una interrelación funcional basada en la cooperación de actividades que abarcan la producción, la promoción, la comercialización y el consumo del producto, este análisis permite identificar los costos en cada eslabón de los competidores claves.

Para conocer el estado mundial de la actividad pesquera la Organización de las Naciones Unidas para la Alimentación y la Agricultura (2014) propone la siguiente metodología:

Captura o extracción: volumen de extracción, precio, artes de pesca utilizada, número de personas a bordo, tiempo de duración de la faena, métodos para conservar el producto hasta el desembarque y participación en asociaciones pesqueras.

Procesamiento: productos procesados (frescos, enlatados, refrigerados o congelados), insumos y costos, número de personas que trabajan en la transformación del producto y tecnologías utilizada.

Logística: costos e importancia del transporte pre y pos-procesamiento, así como las tecnologías utilizadas para asegurar la calidad durante el traslado del producto.

Consumo final: identifican los tipos de productos que venden en los mercados nacionales e internacionales y políticas o regulaciones para el consumo.

Para el análisis de los datos se utilizó la estadística descriptiva al tratarse de un estudio exploratorio de tipo mixto el cual permitió la triangulación de los resultados, demostrándose la consistencia entre las opiniones, mediciones y observaciones realizadas. Esta información 
se clasificó según cada eslabón y se analizó el cambio o modificación que se produjo en la cadena de valor tradicional debido a la incursión del pez León, cuyo alcance se muestra en el Cuadro 1.

\section{CUADRO 1}

Cadena de valor de pesca artesanal en el Caribe sur

\begin{tabular}{ll}
\multicolumn{1}{c}{ Eslabones } & \multicolumn{1}{c}{ Actividades } \\
Captura & $\begin{array}{l}\text { Captura de Pez León con nasas, línea y } \\
\text { buceo a pulmón. }\end{array}$ \\
Comercialización & $\begin{array}{l}\text { Venta de Pez León en restaurantes de } \\
\text { la zona. } \\
\text { Consumo de Pez León por los residentes } \\
\text { de la zona. }\end{array}$ \\
\hline
\end{tabular}

Ética, conflicto de intereses y declaración de financiamiento: los autores declaran haber cumplido con todos los requisitos éticos y legales pertinentes, tanto durante el estudio como en el manuscrito; que no hay conflictos de interés de ningún tipo, y que todas las fuentes financieras se detallan plena y claramente en la sección de agradecimientos. Asimismo, están de acuerdo con la versión editada final del documento. El respectivo documento legal firmado se encuentra en los archivos de la revista.

\section{RESULTADOS}

Se analizaron datos del 2008 al 2015 obtenidos por INCOPESCA, acerca de las capturas de las especies más comunes y de las artes de pesca en el Caribe sur. Las capturas de pargo se realizan con nasa y línea con trozos de macarela como carnada, las capturas de macarela

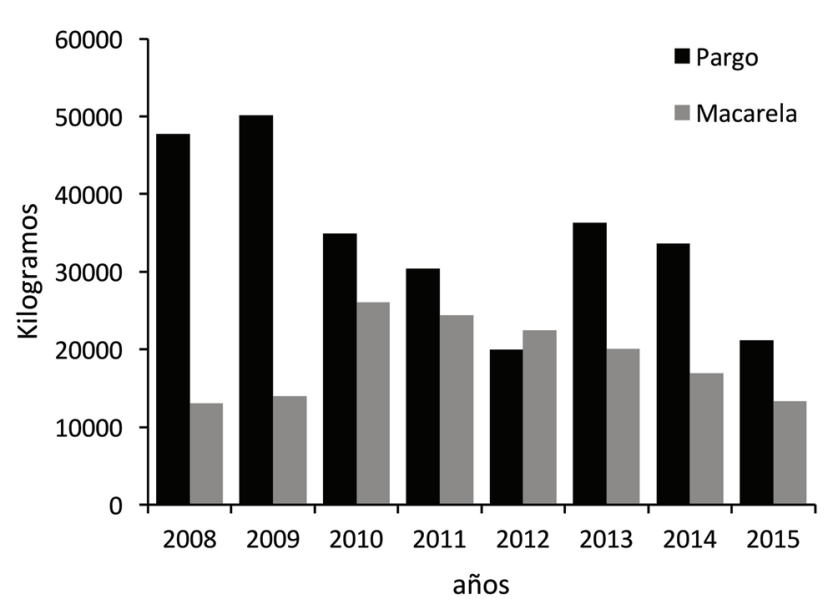

Fig. 2. Capturas de P. pagrus (Sparidae) y S. scombrus (Scombridae), Caribe sur, 2008 - 2015 (Ramos, 2016).

normalmente son con trasmallo, de dichos datos se desprende la afección de las poblaciones de estos peces desde la llegada del pez León en el 2009 (Fig. 2).

Por su parte, las capturas de langosta se realizan con nasas, usando como carnada trozos de coco y macarela y las capturas de camarón normalmente se realizan con atarraya; las capturas de corvina se realizan con línea usando como carnada el camarón (Fig. 3).

Con base en la encuesta a dueños de restaurante sobre productos pesqueros, se obtuvo que los camarones estuvieron disponibles en los restaurantes en un $92 \%$, seguido del Pargo (76\%) y la macarela (74\%), luego la langosta (16\%) y por último la corvina (8\%). Con base en las observaciones a los restaurantes, la comercialización y consumo de estos productos es normalmente de manera frita, en ceviche o en sopa. La mayoría de los restaurantes coinciden en los tres productos más solicitados por los clientes, estos fueron: Pargo, Macarela y camarones.
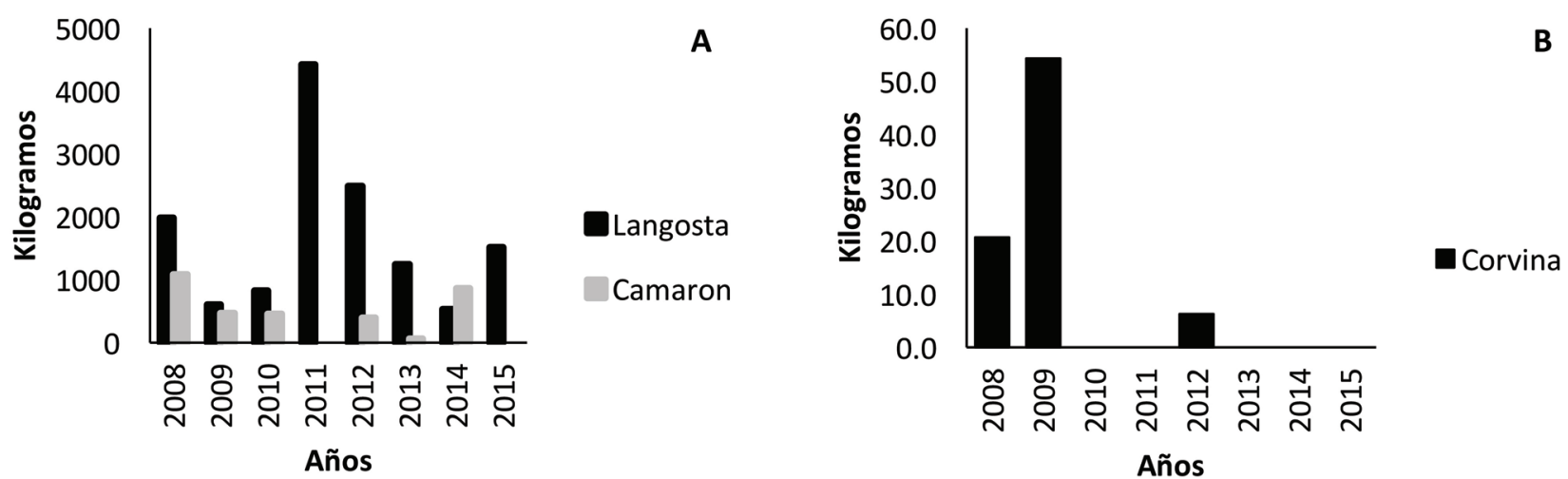

Fig. 3. (A) Capturas de P. argus (Palinuridae) y Camarón (Penaeidae). (B) Capturas de C. albus (Cienidae), Caribe sur, 2008 - 2015 (Ramos, 2016). 
Con las nasas se capturaron 150 individuos, que juntos sumaron $70 \mathrm{~kg}$, con las líneas se pescaron 42 y de estos 18 fueron tiburones incluido un tiburón gata de $3 \mathrm{~m}$, éstos fueron liberados, por lo tanto, el peso con línea sin los tiburones fue de $54 \mathrm{~kg}$. Y del total de la pesquisa no fue posible capturar el pez León con nasa, línea y buceo a pulmón durante el periodo mayo a octubre de 2016.

El Cuadro 2 muestra el total de peces capturados con las nasas y líneas.

CUADRO 2

Número de capturas con Nasas y Líneas

\begin{tabular}{lccc}
\multicolumn{1}{r}{ Nasas } & $\mathrm{N}^{\circ}$ & Líneas & $\mathrm{N}^{\circ}$ \\
Pargo & 90 & Pargo & 18 \\
Pez chancho & 9 & Jurel & 6 \\
Green Jack & 19 & Tiburón & 18 \\
Motín & 1 & Total & 42 \\
Cangrejos & 4 & & \\
Pez Pati & 14 & & \\
Pez Torbi & 5 & & \\
Jurel & 1 & & \\
Pez Ángel & 2 & & \\
Pez Piedra & 1 & & \\
Bobos & 2 & & \\
Platinillas & 2 & \\
Total & 150 & \\
\hline
\end{tabular}

Al paso de recrear la faena de pesca donde se constató la ausencia del pez León en las capturas, las respuestas de los pescadores no están lejos de esta realidad. A continuación, se muestran las respuestas de los pescadores.

Según los datos obtenidos de la entrevista a los pescadores el 30\% lo hacen en la zona de Cieneguita, un 35\% en Cahuita y el 35\% capturan al pez León entre Puerto viejo y Manzanillo.

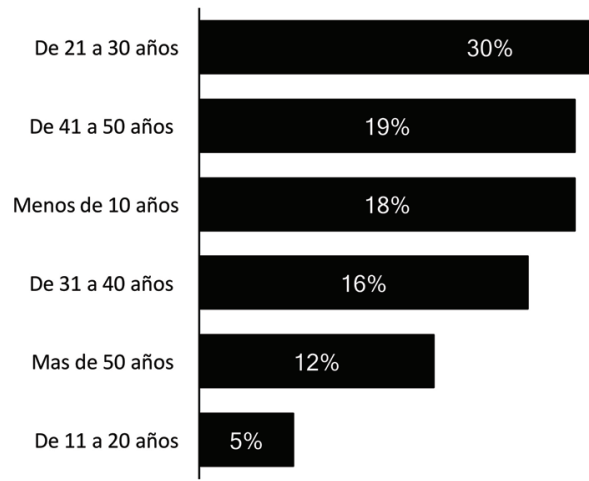

Fig. 4. Tiempo dedicado a la pesca por los pescadores del Caribe sur, 2016.

El 82\% llevan más de diez años pescando (Fig. 4) por lo tanto se han enfrentado a la llegada del pez León en 2009 y han logrado seguir manteniendo su actividad económica pesquera al margen de la especie exótica invasora de la zona Caribe sur.

Según los pescadores el arte de pesca más efectivo para capturar al pez León, fue el buceo a pulmón (Fig. 5), dado la capacidad que tiene el pescador de adentrarse en las cuevas submarinas del arrecife donde se esconde y habita, siendo en las primeras horas de la mañana la mejor captura, debido a su comportamiento inquieto que tiene al buscar su alimento, a lo que hay que sumarle la excelente condición física de los buceadores para descender hasta $15 \mathrm{~m}$ de profundidad.

Los máximos niveles de captura se realizaron entre los periodos del 2010 y 2012 (Fig. 6) donde se dio una considerable sobrepesca del pez León, teniendo un impacto significativo sobre este recurso, debido a que con el paso de los años cada vez se pesca menos, inclusive para 2014, la mayoría de los pescadores registraron su última captura.

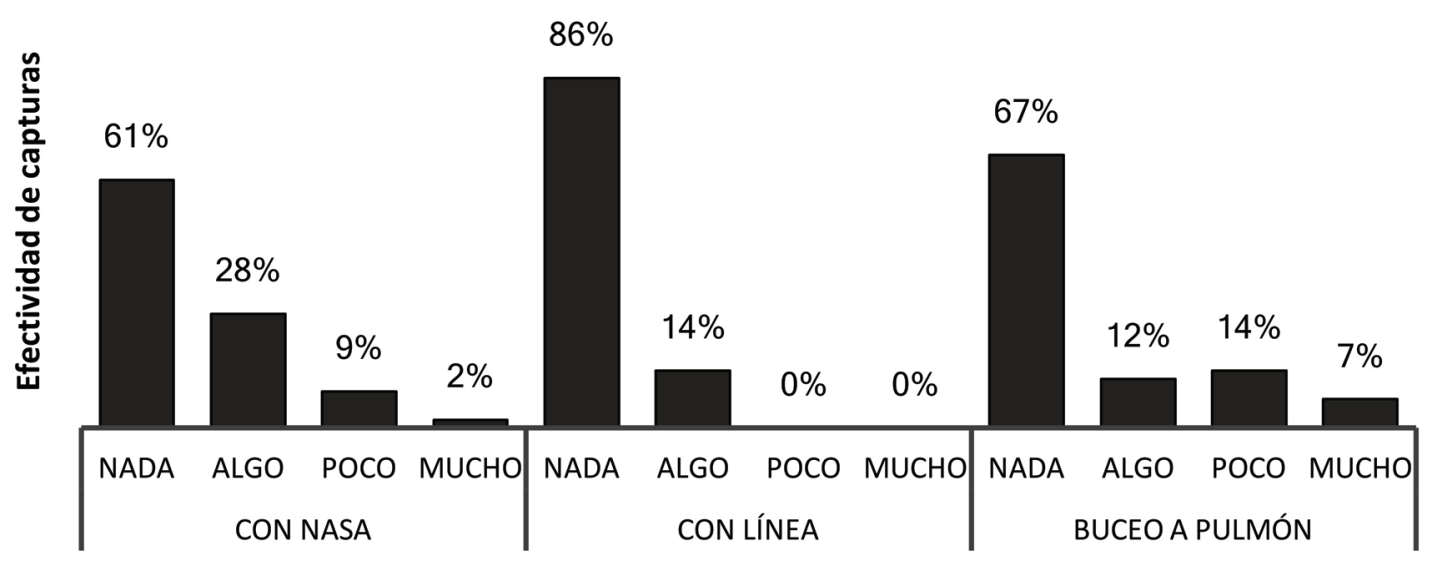

Fig. 5. Capturas de pez León con nasas, líneas y buceo a pulmón, pescadores del Caribe sur, 2016. 
A

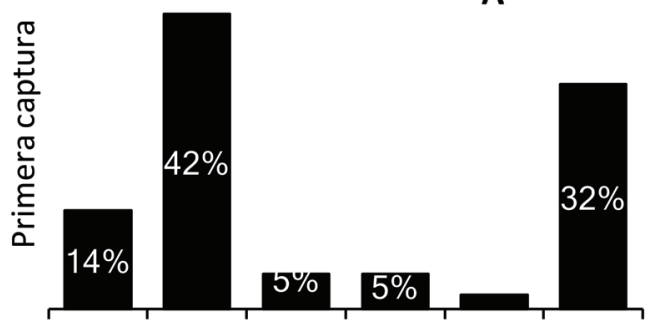

Más de Hace 5 Hace 4 Hace 3 Hace 2 Nunca 6 años años años años años

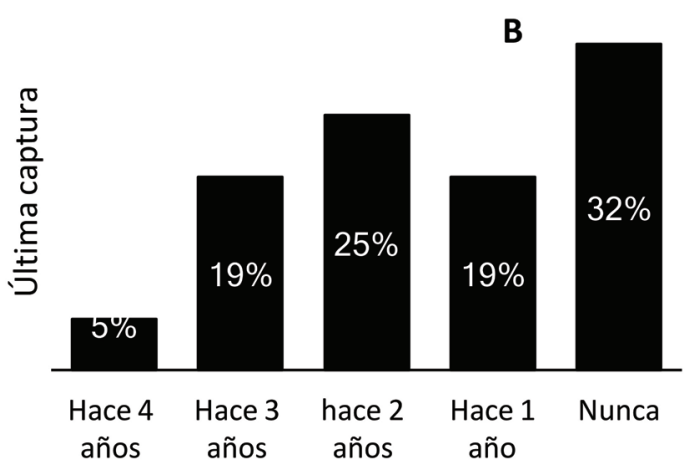

Fig. 6. (A) primera captura. (B) última captura. Historial de capturas de pez León, pescadores Caribe sur, 2016.

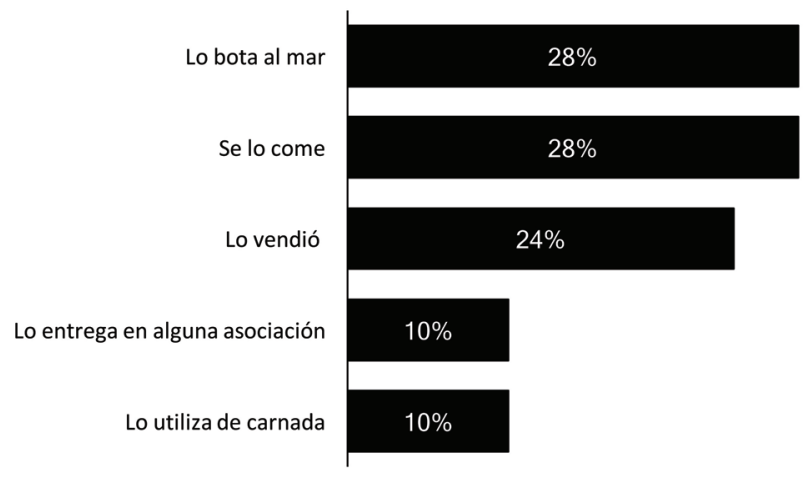

Fig. 7. Usos del pez León Capturado, pescadores Caribe sur, 2016.

Por otra parte, las actividades realizadas por los pescadores muestran que el $72 \%$ de los encuestados le saco algún provecho (Fig. 7), inclusive las asociaciones utilizaron las espinas para hacer joyería.

Además, el $51 \%$ de pescadores no lo consideran como peligroso en su manipulación (Fig. 8).

En el Cuadro 3 se muestra el precio de estos productos en ASPELTA.

CUADRO 3

Lista de precios

\begin{tabular}{cc} 
Producto & Precio $(\$ / \mathrm{k})$ \\
Pargo & 7,5 \\
Macarela & 5,8 \\
Langosta & 13,3 \\
Camaron & 11,6 \\
Corvina & 7,5 \\
Pez León & 8,3 \\
\hline
\end{tabular}

Por otra parte, durante la visita al centro de acopio de Cieneguita no tenían oferta del pez León. Tampoco

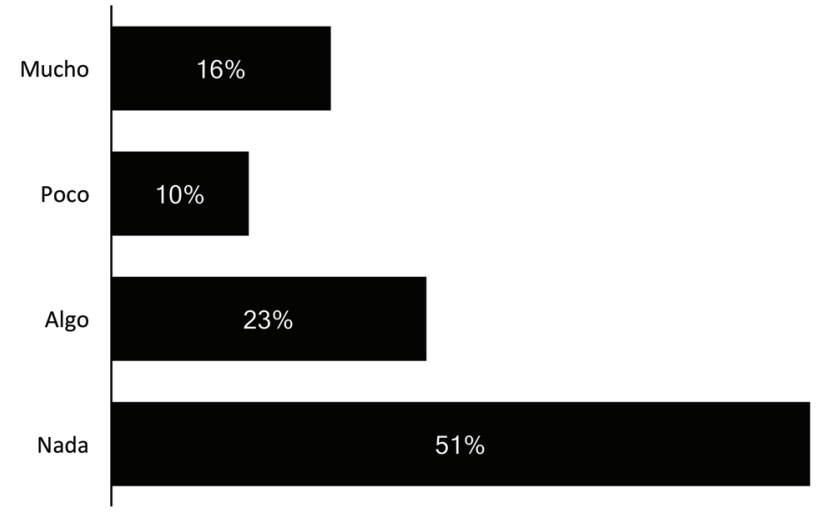

Fig. 8. Riesgo en la manipulación del pez León, Caribe sur, 2016.

se han realizado adaptaciones en las trampas para aumentar su captura. El administrador Parkins de ASPELTA destaca que "entre el 2010 y 2012, se capturaba mucho en las nasas que tenían como carnada macarela y coco y que este a su vez se comía los peces que quedaban atrapados dentro de la jaula, y todos los meses se capturaba. Uno de los problemas con la contabilización de capturas con las nasas, es que no se puede cuantificar cuanto y que pescados se ha comido el pez León capturado. Hacia finales del 2013 comenzaron a descender las capturas al paso que en 2016 capturaron solamente uno de casi $1 / 2 \mathrm{~kg}$ con el arte de pesca Línea a una milla en dirección Noroeste del centro de acopio de Cieneguita, cerca del muelle de JAPDEVA".

Uno de los informantes de ASPELTA considera que "es probable que las poblaciones estén concentradas en el muelle de JAPDEVA, ya que en este lugar no pueden ingresar los pescadores y a su vez hay una importante perturbación del medio acuático debido a la permanente entrada y salida de grandes barcos, por lo que tradicionalmente se le ha catalogado como una zona contaminada y sin mayor diversidad de peces". 
Por su parte, tanto los pescadores de Cahuita como los de Manzanillo han comentado su interés por construir nasas de color oscuro para intentar despertar el interés del pez León por adentrarse en esta jaula debido a su comportamiento críptico, a su vez manifestaron el deseo de construirlas con forma redondeada y con un tamaño tres veces menor a las nasas utilizadas en esta investigación (que son las que normalmente se usan) con el fin de evitar dañar el coral lo menos posible, sin embargo son de mayor costo y la problemática que viven los pescadores por el robo de nasas reduce el interés en invertir este otro tipo de nasa y por lo tanto no fue posible constatar su construcción durante esta investigación.

Los pescadores de Manzanillo realizaron experimentos usando las hormonas del pez León, colocándolas junto con la carnada dentro de las nasas y comprobando si con esta técnica se atraen más hembras, o bien, mayor cantidad de peces en general. Esta técnica fue enseñada por los especialistas del INA, Talamanca; sin embargo, el experimento no atrajo al pez León y lo que se capturó fue al pez chancho, conocido como viejas.

Por otra parte, con base en las entrevistas realizadas a los dueños de restaurante, distribuidos a lo largo de la costa Caribe sur, siendo Cahuita la zona en la que hay más restaurantes (Fig. 9). Se entrevistó a: propietarios, administradores y empleados, de los cuales, el 15\% asegura que al menos en una ocasión los clientes han solicitado platillos a base de pez León; sin embargo, solamente el 3\% hizo un cambio en su oferta gastronómica al incorporarlo dentro del menú; el pez León se prepara: frito, acompañado con patacones, en ensalada, en arroz, en sopas y otras opciones. En otras palabras el $97 \%$ de los restaurantes no ha realizado un cambio en su oferta gastronomica, para introducir al pez León.

El restaurante "Monli Restaurant", ubicado en el centro de Puerto Viejo, es reconocido por vender platillos de

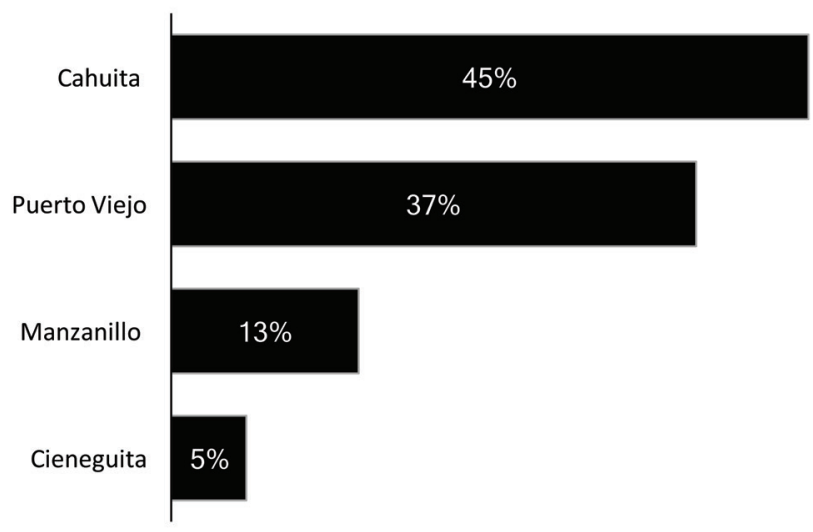

Fig. 9. Concentración de los restaurantes con relación a la localidad, Caribe sur, 2016. pez León. Pero, el día que se les visitó se solicitaron cuatro platos y solo tenían dos, lo que indica que no obtuvieron los beneficios económicos esperados, ya que fueron condicionados por la baja cantidad de materia prima disponible. Lo anterior, no les permitió obtener mayores ganancias demostrandose la escasez, dificil acceso, poca pro actividad o interés de los restaurantes y pescadores en atender la demanda de este producto.

Este ciclo de cadena de valor, beneficia a su vez a cada eslabón, ya que el pescador obtiene una ganancia de 5 000 colones por cada kilo, el restaurante obtiene 4000 colones de ganancia por su preparación y comercializacion; siendo el precio final en el mercado para su consumo de 9000 colones en adelante.

Con base en los residentes encuestados, solamente un $10 \%$ lo ha consumido, $7 \%$ de ellos viven en Puerto Viejo y el resto en Cahuita, las personas de Cahuita que lo han consumido lo han hecho en el restaurante Monli en Puerto Viejo. Estas personas lo consumieron frito y consideraron que el precio estaba muy elevado, por lo tanto, lo han consumido pocas veces y en algunos casos solo una vez a manera de curiosidad hace algunos años, todos afirmaron que tiene buen sabor. Al consultarles a las personas que no lo han consumido, por qué no lo han hecho, las respuestas más frecuentes fueron: porque nadie lo vende, porque es venenoso o porque es muy caro. Es decir, el $90 \%$ de los residentes no han cambiado su dieta o lo han incorporado.

\section{DISCUSIÓN}

Las especies que se han venido comercializando, presentan un comportamiento estable, su comercialización es apreciable en los menús de los restaurantes visitados de la zona, durante esta investigación, de los cuales, sus platillos son a su vez la base de los concursos de comidas caribeñas y de festivales como el de la "Diáspora Africana", celebrado todos los años a finales de agosto, donde se exhibe la cultura caribeña propia, su gastronomía entre otros, por lo que este recurso se ha logrado mantener con el tiempo hasta llegar a ser la dieta normal en el consumo del limonense.

El P. Pagrus, comunmente llamado pargo, por la cantidad, su estado de conservación es considerado de preocupación menor [Ic] (UICN, 2014). Sin embargo; presenta una tendencia a la baja, debido a que, en el 2008 se reportaban $50000 \mathrm{~kg}$ y para el 2015 se reportaton 30 $000 \mathrm{~kg}$ y por su categoría de preocupación menor, según la UICN (2014), se considera que no se da la sobre pesca de este recurso. Otra posibilidad poco probable, es que los datos no estén debidamente actualizados. A lo 
anterior se une la suposición de que es el pez León, esté afectando las poblaciones de pargo, ya que de acuerdo con Sandel (2011) el pargo figura de segundo lugar de importancia en su dieta.

Por otra parte, los datos de la especie S. Scombrus, comunmente llamada macarela, muestran un estado de conservación considerado de preocupación menor [lc] (UICN, 2014) y en el Caribe sur se encuentra estable. Los peneidos, conocidos como camarón, con un estado de conservación vulnerable [vu] (UICN, 2014), muestran que se han mantenido sus poblaciones en equilibrio, a pesar de que se presentan picos de disminución en algunos años, es decir, que hubo sobre pesca y dado que en algún tiempo no han ocurrido capturas se logró regenerar el recurso (Ramos, 2016).

Para la especie Panulirus argus conocida como langosta espinosa, la UICN ha considerado que no hay datos suficientes [dd] para catalogar su estado de conservación, ya que hay lagunas en los datos de pesca o en otros índices adecuados para determinar un índice de abundancia relativa apropiada para esta especie y en que medida, se ve afectada su cosecha debida a amenazas del medio donde se distribuye, con más información sobre la captura por unidad de esfuerzo, podría considerarse dentro de la categoría de amenaza (UICN, 2014).

La especie Cynoscion albus, conocida por su nombre común corvina, también presenta un estado deficiente de datos [dd] (UICN, 2014) para catalogar su estado de conservación, debido a que se necesita más información sobre el impacto de la pesca en su población, la cual está correlacionado con los datos que maneja el INCOPESCA, considerando que a partir del 2013, no registran datos y como señala Galarza y Kamiche (2014) hay pescadores sin los derechos de propiedad (licencia de pesca) éstos no brindan el dato, y como se anotó anteriormente, este producto está disponible en el centro de acopio de Cieneguita (Parkins, 2015).

La poca captura de pez León hoy en día, se podría deber a que en los últimos años existió una sobre pesca considerable, especialmente entre el 2010 y el 2014, o en su efecto que estén a grandes profundidades o en zonas no visitadas por los buzos. El administrador del centro de acopio de Cieneguita y los pescadores entrevistados señalan que en años pasados cada mes capturaban importantes cantidades de pez León, precisamente cuando comenzaba a establecerse en este ecosistema marino, lo cual, al no observarse tanto pez León durante el estudio, podría ser un indicador de ampliación de sus nichos a otros sitios. Además, se resalta lo señalado por Aguilar (2002) cuando la tasa de extracción es superior a la tasa de crecimiento natural se vuelve insostenible la actividad, en consecuencia, escaseando el recurso y manteniéndose bajas las poblaciones de esta especie en este ecosistema marino.

Del mismo modo, Smith (2015) (citado en ODISEA, 2015), destaca que el pez León, tiene un crecimiento natural, de seis meses, que es lo que tarda en repoblar una zona que habían dejado limpia, sin embargo; los pescadores lo capturaron mensualmente, evidenciando la presión ejercida, ya que estas capturas se dieron por encima de su crecimiento natural.

Por otra parte, cuando los pescadores salen al mar utilizando sus artes de pesca (nasas y líneas), no salen a capturar solo al pez León, ellos conocen las dificultades que hay para capturarlo. Sin embargo; de acuerdo con Sandel (2011), quien ha documentado poblaciones establecidas del pez León a lo largo del Caribe sur y considerando que al no haber un control absoluto del medio marino como lo señalan Galarza y Kamiche (2014), puede existir al menos una leve posibilidad, de quedar capturado con estas artes de pesca como lo confirman algunos pescadores que capturaron con Nasas y Líneas al pez León durante el período 2010 y 2014, sin realizar ninguna modificación a estas artes de pesca.

Para la mayoría de los pescadores artesanales el pez León no representa ningún peligro a la hora de prepararlo, el peligro es que presenta una glándula que expulsa toxinas que contienen acetilcolina, una toxina neurotóxica que se considera veneno porque afecta la neurotransmisión, ubicada en la base de sus 18 aletas dorsales o espinas punzantes, en las anales y las pélvicas, pero ellos consideran que esto no influye en la actividad pesquera. La baja y casi nula comercialización de este producto por parte de los pescadores se debe principalmente a la escaza cantidad que se registra hoy en día en el Caribe sur de Costa Rica, sumado al gran esfuerzo que se debe realizar para su captura lo que ha generado una pérdida de interés de algunos pescadores para trabajar este producto.

Por lo anteriormente expuesto, se puede asumir que, con la incursión del pez León no se ha evidenciado un cambio en el tipo de pesca artesanal en el Caribe sur, se mantiene relativamente constante la frecuencia de pesca en cuanto a: los viajes, los sitios, las artes de pesca, especies blanco, y en cuanto a los volúmenes de pesca se requiere más investigación sobre factores bióticos y abióticos. Es importante señalar que para determinar los efectos directos del pez León sobre las especies de interés comercial del Caribe se requieren hacer estudios más detallados entorno a dichas especies y su relación con la presencia y/o ausencia de esta especie exótica, por ello la relación causal no se puede determinar debido a que el objetivo del estudio no aborda la misma. 
A su vez, el ecosistema marino del Caribe sur, incluye áreas marinas protegidas que se destacan por su riqueza marina, que se traduce en un ambiente marino con grandes depredadores como el tiburón, mero y barracudas que posiblemente ya comenzaron a consumir al pez León, contribuyendo a mantener bajas las poblaciones, al igual que en otros sitios como en las Bahamas e Islas Caimán, donde García (2015), reporta que los depredadores nativos como la Chema criolla (Epinephelus striatus) y el tiburón gata (Ginglymotoma cirratrum), son depredadores del Pez León. A su vez, Mumby, Harborne y Brumbaugh (2011), reporta que en las Bahamas su densidad y biomasa son menores en los sitios donde la abundancia de meros es mayor, especialmente en las profundidades adonde no llegan los buzos.

De acuerdo con los pescadores encuestados, el arte de pesca más eficaz para capturarlo es el buceo a pulmón, con el que se puede llegar a las cuevas y lugares selectos que utiliza el pez León para camuflarse. Esto representa un importante esfuerzo pesquero por cada unidad capturada, lo cual deberá influir en que se le dé un valor superior en el mercado. Por el contrario, a lo que ha sucedido en otros países, por ejemplo, en las Islas Caimán, donde su comercialización, ha incidido de forma relevante en las prácticas locales, que incluso han incentivado a cultivar, cosechar y comercializar el pez León para la exportación (Johnson \& McCoy, 2015). Sin embargo, este tipo de comercialización es prohibida en Costa Rica (Poder Ejecutivo, 2016).

Los pocos restaurantes que hicieron el cambio obtuvieron un valor adicional, ya que se considera a la carne del pez León, como un producto diferenciado, con un impacto positivo, tal como lo mencionan Quintero y Sánchez (2006), en cuanto más diferente sea un nuevo producto en el mercado, dicho producto podrá tener mayor poder sobre los demás productos establecidos, lo que se traduce en mayores beneficios económicos y en un mercado más competitivo. El beneficio para los dueños de los restaurantes se ve reflejado en una oferta gastronómica más variada y para los pescadores en un mayor valor agregado para su pesca. Para el consumidor al ser una carne blanca puede ser preparado en diversos platos como ceviche, pecado frito, dedos de pescado, en pasta, empanadas, etc.

Continuando con lo expresado en el párrafo anterior, se observa que la influencia del pez León en la cadena de valor, es muy baja, debido a que los potenciales consumidores de esta región Caribe sur, aún desconocen el sabor, nutrición y seguridad. Esto se analizó ante una mayoría de respuestas obtenidas de los residentes encuestados, que mencionaron no haberlo consumido, algunos porque creen que es venenoso, otros porque no saben cómo conseguirlo, o los que no lo han consumido porque lo consideran muy caro. Esto debe activar las alarmas para que la Comisión Nacional para el Manejo, Control y Aprovechamiento del pez León en Costa Rica pueda valorar y mejorar la utilidad que se le da a este recurso, falta capacitación y encadenamientos para que el pez León sea realmente aprovechado sosteniblemente.

Además, llama la atención que un $28 \%$ de los pescadores que capturaron el pez León en años anteriores con nasa y con línea afirmaron haberlo tirado al mar sin sacarle un mejor provecho, tan solo el $24 \%$ de pescadores lo comercializó, siendo una minoría los que obtuvieron ganancias por sus capturas. La baja comercialización y conocimiento del producto que hay, hacen que su influencia sea moderada sobre los demás productos, al no existir materia prima disponible para ingresar al mercado y competir, la mayoría de los restaurantes, no han realizado cambios en su oferta gastronómica, estos cambios están siendo condicionados por la insuficiente cantidad de materia prima, lo cual se refleja en su comercialización en pocos restaurantes (3\%), pudiendo comercializar el producto solo a nivel local.

Así las cosas, el mercado que tiene el pez León en Costa Rica, es de un uso muy limitado, ya que, está siendo destinado a un sector social específico; principalmente clase media y turistas, quienes lo consumen por curiosidad y por consiguiente, la mayoría de los consumidores residentes de la zona no lo han incorporado en su dieta. La manera más común de consumo del pez León, ha sido, prepararlo: en ceviche, frito y acompañado de patacones, con arroz y ensalada, entre otros. Con un precio superior a los otros productos establecidos de la cadena de valor de pesca artesanal. Demostrándose otro aspecto relevante que señala Quintero y Sánchez (2006) sobre el precio el cual es condicionado si el cliente está dispuesto a pagar a un precio mayor que los costos por unidad producida de otros productos establecidos de la cadena de valor.

A su vez, el desconocimiento que tienen los pobladores sobre el pez León, ha generado un mayor esfuerzo para capturarlo hasta acabar con los mismos y posiblemente se ha dado una sobre pesca que redujo considerablemente estas poblaciones, según lo planeado por la gobernanza. A lo que cabe recordar lo que dice Aguilar (2002), al ser un capital natural renovable la extracción debe ser cuidadosa, particularmente porque afectará los periodos posteriores, es decir es un recurso que no se puede extraer ilimitadamente.

Sin embargo, datos de los locales arrojan que en los alrededores del muelle de JAPDEVA e Isla Uvita, se registra 
una población de pez León, sitios donde no hay presión pesquera (Parkins, 2015), a su vez, también se logró establecer en el Refugio de Vida Silvestre Manzanillo sitio RAMSAR, lugar que, dado a su categoría de protección, influye para que no se dé una gran captura. En esta zona de Manzanillo, es en la que se realizan los torneos de Pez León, donde coincidió con esta investigación y hubo poca captura de dicho pez, comparado con los torneos realizados en el mismo lugar en años anteriores, con lo cual, al no haber una fuerte presión pesquera, es posible que los depredadores estén influyendo en las bajas poblaciones de este sitio, o también se puede deber a condiciones oceanográficas que podrían ocasionar el movimiento de la especie a otros sectores, por tal motivo no se puede señalar que dicha ausencia se debe a un único factor porque podría ser multifactorial.

Durante el torneo de Pez León en el 2016 hubo concursantes que no lograron capturar nada, ya que solo fueron capturados 38 individuos en 3 horas entre 50 participantes y algunos capturaron más de dos individuos, esto representa un esfuerzo de 150 horas lo que refleja la disminución y poca cantidad del Pez León que hay en este ecosistema marino y la gran cantidad de esfuerzo que representa para capturarlo, estos datos concuerdan con los obtenidos durante la faena de captura de esta investigación.

Además, durante años anteriores, los pescadores entrevistados señalaron, que de igual manera el Pez León, quedaba capturado con las nasas tradicionales. A su vez, según el decreto ejecutivo № 39627 para la creación de la Comisión Nacional para el Manejo, Control y Aprovechamiento del pez León en Costa Rica, se considera en su inciso seis, que está siendo capturado por el $50 \%$ de las nasas utilizadas por los pescadores artesanales del Caribe sur (Poder Ejecutivo, 2016), por tanto, se considera que la nasa es efectiva para capturarlo indistintamente del color y el que no quedaran capturados, se pudo deber a que no estaba presente en los sitios muestreados durante la investigación.

Conforme se desplaza la pesca hacia el Sureste, se reducen los espacios de pesca, debido a la presencia de áreas marinas protegidas, y que históricamente se ha complementado la pesca con actividades para el Turismo. El contexto sitúa un incremento de actividad pesquera en sitios de arrecife, con la justificación de matar al pez León, por lo que se debe tener un gran cuidado, ya que las nasas pueden destruir importantes estructuras de arrecife que tarda miles de años en su construcción y que albergan una gran biodiversidad marina y Carbono (Burke y Maidens, 2005) por lo anterior, solamente se debería pescar en estos sitios con buceo a pulmón. Sin embargo, el fenómeno motivo a la Gobernanza a situar un contexto de mayor pesca en los sitios de arrecife, inclusive hubo importantes donaciones de recursos para motivar la construcción de artes de pesca y capturarlo cerca de estas áreas.

Un mecanismo de respuesta con una visión deł nativismo, el cual considera que la naturaleza es frágil y vulnerables a la influencia destructiva de los seres humanos, por lo tanto, se debe proteger y preservar su integridad biológica en equilibrio intentando restaurar la naturaleza y que se asemeje lo más posible a la "estructura original" de un paisaje prístino, donde los indeseables en manos de la gestión son necesarios para evitar su introducción o erradicarlas (Carballo, 2015).

A su vez, no hay evidencia de que hay mucha población de pez León, para predecir si este pez genera un impacto irrelevante o significativo en un ecosistema marino, se creó en Belice, el modelo de Ann y Valderrama (2015), quienes calcularon la tasa de producción de biomasa del pez León, con la tasa de producción de biomasa de los peces que fueron su presa, la productividad local de las poblaciones de peces presa, resultó lo suficientemente alta como para compensar la depredación y pesca del pez León. Se recomendó, que la densidad del pez León, no supere los 32 peces/ ha. Ya que los costos pesqueros involucrados en alcanzar las densidades objetivo compensan la viabilidad económica de las técnicas de remoción manual, como una estrategia de manejo a largo plazo para las poblaciones invasoras.

De momento, con el estudio anteriormente citado donde 32 peces/ ha se considera un impacto irrelevante en Belice y con relación al estudio de Sandel (2013) donde contabilizó una población de 91 peces / ha para el Caribe sur, parece justificar la necesidad de la captura por el ser humano, sin embargo; hace falta determinar cuál sería el número de pez León que impactaría negativamente los ecosistemas marinos del Caribe costarricense, esto para definir y tomar decisiones de manejo de la especie. A su vez, para actualizar la tasa de población anualmente a fin de encaminar las acciones de barrido.

\section{AGRADECIMIENTOS}

Agradecemos al proyecto de investigación titulado "Impactos biológicos, sociales y económicos a causa de la especie introducida pez León ( $P$. Volitans / Miles), en la región Caribe de Costa Rica" ejecutado por la Universidad Nacional y la Universidad Estatal a Distancia y que cuenta con financiamiento FEES-CONARE 


\section{REFERENCIAS}

Aguilar, B. (2002). Paradigmas económicos y desarrollo sostenible: la economía al servicio de la conservación. San José, CR: EUNED.

Albins, M., \& Hixon, M. (2008). Invasive Indo - Pacific lionfish Pterois volitans reduce recruitment of Atlantic coral - reef fishes. Marine Ecology Progress Series, 367, 233-238. DOI:10.3354/meps07620

Álvarez, Y. (2014). Cocina tradicional costarricense 6: Limón. San José, Costa Rica: Ministerio de Cultura y Juventud. Centro de investigación y conservación del Patrimonio Cultural. Instituto Costarricense de Turismo.

Ann, K., \& Valderrama, D. (2015). How Many Lionfish are Too Many Lionfish? Estimating Target Densities and Costs Associated with Manual Removal of Invasive Lionfish Stocks in a Marine Reserve in Belize. In Book of Abstracts. $68^{\text {th }}$ Annual Gulf and Caribbean Fisheries Institute. (2015). Panamá: MarViva.

Burke, L. \& Maidens, J. (2004). Reefs at risk in the Caribbean. Washington, D.C., EE.UU.: World Resources Institute.

Carballo, E. (2015). Controversies and consensus on the lionfish invasion in the Western Atlantic Ocean. Ecology and Society, 20(3), 24. DOI:10.5751/ES-07726-200324

Galarza, E., \& Kamiche, J. (2015). Pesca artesanal: Una oportunidad para el desarrollo. Lima, Perú: Universidad del Pacífico. Recuperado de http://agenda2014.pe/ publicaciones/agenda2014-pesca-artesanal.pdf. DOI:10.21678/978-9972-57-342-2

García, A. (2015). Principales relaciones ecológicas del pez León (Pteoris volitans/miles) en arrecifes de La Habana, Cuba (Tesis de Maestría). Centro de Investigaciones Marinas, Universidad de La Habana, Cuba.

Green, S., Akins, J., Maljković, A., \& Côté, I. (2012). Invasive lionfish drive Atlantic coral reef fish declines. PLoS One, 7(3), e32596. DOI: 10.1371/journal.pone.0032596

Hernández, A. (2015). Entrevista de R. Ríos [archivo de audio]. Pescador Caribe sur, Punta Uva. Recuperado de https:// entrevistaspezleoncostarica.blogspot.com/2019/08/ entrevistas-para-pescadores-artesanales.html

Johnson, B., \& McCoy, C. (2015). Lionfish Control and Management in the Cayman Islands: Seven Years On, Lessons Learned. In Book of Abstracts. $68^{\text {th }}$ Annual Gulf and Caribbean Fisheries Institute. (2015). Panamá: MarViva.

Karp, P., Chapman, J., Fruitema, M., Curtis, J., Saul, N., \& Spencer, E. (2015). Incentivising Lionfish Removals Through Development of Markets for Jewelry: Preliminary Experiences from Belize, the Bahamas and St. Vincent and the Grenadines. In Book of Abstracts. $68^{\text {th }}$ Annual Gulf and Caribbean Fisheries Institute. (2015). Panamá: MarViva.

Lesser, M. \& Slattery, M. (2011). Phase shift to algal dominated communities at mesophotic depths associated with lionfish (Pterois volitans) invasion on a Bahamian coral reef. Biological Invasions, 13(8), 1855-1868. DOI: 10.1007/ s10530-011-0005-z

Lid, D. Marchal, W., \& Wathen, S. (2012). Estadística aplicada a los negocios y la economía (15ed). México D.F., México: Mc Graw Hill Education.

Mairena, M. (2015). Entrevista de R. Ríos [archivo de audio]. Influencia del Pez León en la pesca artesanal. Pescador Caribe sur. Cahuita. Recuperado de https://entrevistaspezleoncostarica.blogspot.com/2019/08/entrevistas-para-pescadores-artesanales_9.html

McDonald, H. (2015). Entrevista de R. Ríos [archivo de audio]. Pescador Caribe sur, Puerto Viejo. Recuperado de https:// entrevistaspezleoncostarica.blogspot.com/2019/08/1. html

Morris, J. (2013). El Pez León Invasor: guía para su control y manejo. Florida, USA: Gulf and Caribbean Fisheries Institute.

Mumby, J., Harborne, A., \& Brumbaugh, D. (2011). Grouper as a Natural Biocontrol of Invasive Lionfish. PLOS ONE, 6(6), 1-4. DOI: 10.1371/journal.pone.0021510

Odisea. (Productor). (2015). Los invasores más letales del mundo, el Pez León gigante [video]. Recuperado de http://www.documentalesgratis.es/ invasores-mas-letales-mundo-pez-leon-gigante.

Organización de las Naciones Unidas para la Alimentación y la Agricultura. (2014). El estado mundial de la pesca y la acuicultura: Oportunidades y desafíos. Roma, Italia. Recuperado de http://www.fao.org/3/a-i3720s.pdf.

Parkins, J. (2015). Entrevista de R. Ríos [archivo de audio]. Administrador ASPELTA, Cieneguita. Recuperado de https://entrevistaspezleoncostarica.blogspot. com/2019/08/entrevistas-para-pescadores-artesanales_10.html

Parques Nacionales de Costa Rica. (2016). Gandoca Manzanillo. Área de conservación la Amistad Caribe. Recuperado de: http://costaricaparquesnacionales.com/Areas/ ACLAC/gandoca/index.htm

Poder Ejecutivo. (2016). Creación de la Comisión Nacional para el Manejo, Control y Aprovechamiento del Pez León en Costa Rica, decreto Ejecutivo N 39627. San José, Costa Rica: Procuraduría General de la Republica

Porter, M. (2005). Estrategia competitiva. Técnicas para el análisis de los sectores industriales y de la competencia (1.st ed.). México, D.F., México: Continental.

Quintero, J. \& Sánchez, J. (2006). La cadena de valor: Una herramienta del pensamiento estratégico. Maracaibo, Venezuela: Universidad Privada Dr. Rafael Belloso Chacín.

Ramos, G. (2016). Correo electrónico de M. Laguna [archivo de texto]. Funcionario INCOPESCA, Limón. Recuperado de https://entrevistaspezleoncostarica.blogspot. com/2019/08/pesca-total-segun-clasificacion.html 
Ríos, R. (2015). Entrevista de M. Laguna [archivo de audio]. Sociólogo, Vicerrectoria de investigación, UNED, Sabanilla. Recuperado de https://entrevistaspezleoncostarica.blogspot.com/2019/08/impactos-biologicos-sociales-y.html

Sandel, V. (2011). El pez León (Pterois volitans/miles complex) en el Área de Conservación La Amistad - Caribe, Costa Rica estado actual de la población invasiva y percepciones para su manejo (Tesis de Maestría). Universidad Nacional, Heredia, Costa Rica.
Sistema Nacional de Áreas de Conservación [SINAC] (2016). Parque Nacional Cahuita. Recuperado de: http://www. sinac.go.cr/ES/ac/aclac/pnc/Paginas/default.aspx

UICN (Unión Internacional para la Conservación de la Naturaleza). (2014). The UICN red list of Threatened Species. U.K. recuperado de http://www.iucnredlist.org/

Ustate, E. (2005). Diagnóstico de la cadena productiva pesquera en la República de Colombia. Ministerio de Comercio, Industria y Turismo: UNIDO. Recuperado de: http://www.unido.org/fileadmin/import/24117_ DiagnsticoNacionaldeColombia.pdf 